\title{
Conocimientos financieros y capacidades financieras en colaboradores de una organización del Noreste Colombiano
}

\author{
Financial knowledge and financial capabilities in employees of an organization in \\ Northeast Colombia
}

\author{
César Augusto Saldarriaga Restrepo ${ }^{1}$ \\ ${ }^{1}$ Escuela Profesional Contabilidad, Facultad de Ciencias Empresariales, Universidad Peruana Unión
}

INFORMACIÓN DEL ARTÍCULO

Historia del artículo

Recibido: 08 de octubre 2017

Aceptado: 03 de mayo 2018

Palabras clave:
Conocimientos financieros,
Capacidades financieras,
$\begin{aligned} & \text { Educación financiera, Ahorro, } \\ & \text { Presupuesto. }\end{aligned}$

Palabras clave:

Educación

Presupuesto.

\section{Resumen}

A nivel mundial la Organización para la Cooperación y el Desarrollo Económico (OCDE), promueve la educación financiera con la finalidad de desarrollar conocimientos económicos en los ciudadanos para valerse en la vida, entender la sociedad y mejorar el mundo, de allí que, el estudio determina en qué medida los conocimientos financieros de los colaboradores de una organización del Noreste Colombiano se relacionan con sus capacidades financieras, como aspecto clave de la administración financiera. Para ello, se estableció un diseño cuantitativo descriptivo y correlacional no experimental de corte transversal, en la que se incluyeron por muestra probabilística 114 colaboradores, y se aplicó la encuesta sobre conocimientos y capacidades financieras diseñada para esta investigación y validada con un Alfa de Cronbach de 0.876; mostrando una alta consistencia interna. Además, se realizaron preguntas sociodemográficas y complementarias incluidas en la misma encuesta. Los resultados evidencian una relación significativa del $47 \%(0.476)$ y valor $(P<0.000)$, entre los conocimientos financieros y las capacidades financieras de los colaboradores. A nivel de resultados complementarios se encontró que las mujeres se endeudan más que los hombres y el conocimiento financiero es la variable con mayor dificultad para la población. Asimismo, las capacidades financieras se manifiestan con un $62 \%$ en un nivel medio alto y alto como fortaleza de la población. Por tanto, siendo que las variables se relacionan directa y positivamente, se deben potenciar estrategias para mejorar la administración de recursos en los empleados por medio de la construcción de escenarios de formación y educación financiera.

\begin{abstract}
Worldwide, the Organization for Economic Cooperation and Development (OECD) promotes financial education with the purpose of developing economic knowledge in citizens to use in their lives, understand society and improve the world. With this in mind, this study determines the extent to which the financial knowledge of the collaborators of an organization in Northeast Colombia is related to their financial capabilities, as a key aspect of financial administration. For this, a descriptive and correlatio-
\end{abstract}




\section{Keywords:}

Financial knowledge, Financial capabilities, Financial education, Saving, Budget. nal non-experimental quantitative design using a cross-section was established, in which 114 collaborators were included by probabilistic sample, and they were given survey on financial knowledge and capabilities designed for this research study and validated with a Cronbach's Alpha of 0.876 , showing high internal consistency. In addition, socio-demographic and complementary questions were included in the same survey. The results show a significant relationship of $47 \%(0.476)$ and value $(P<0.000)$ between the financial knowledge and the financial capabilities of the employees. At the level of complementary results, it was found that women borrow more than men and financial knowledge is the variable with the greatest difficulty for the population. Likewise, financial capacities are manifested in $62 \%$ at a medium high and high level as a strength of the population. Therefore, since the variables are directly and positively related, strategies should be promoted to improve the administration of resources in employees through the construction of training and financial education scenarios.

\section{Introducción}

La economía global gira en torno a la adquisición, el uso y la acumulación de recursos. Las personas constantemente se enfrentan a hechos que implican el uso del dinero. Se podría decir que la mayoría de las decisiones que se toman tienen implicaciones económicas, esto hace que cada persona sienta la necesidad de obtener ingresos para sufragar los gastos y que de acuerdo con el nivel de conciencia y en función a las aspiraciones individuales que deseen, es a saber, ahorrar, endeudarse, invertir, hacer provisiones para necesidades futuras o simplemente acumular riquezas, entre otras.

Gracias a los hallazgos visibilizados de diferentes estudios, se ha identificado la importancia de contar con conocimientos financieros y de desarrollar capacidades financieras que permitan manejar los riesgos, aprovechar los beneficios que las actividades financieras envuelven y alinear las decisiones financieras con los objetivos personales o familiares de largo y mediano plazo se convierte en fundamental. La falta de conocimientos financieros incapacita a los individuos para analizar la información financiera disponible y las escasas capacidades financieras los limitan para reaccionar ante dicha información.

Pese a que la educación financiera se ha puesto de moda, el trabajo de los padres, la escuela, la iglesia, los gobiernos y las entidades financieras solo se puede limitar a impartir conocimiento financiero teórico; con el deseo de que estos sirvan de fundamento para que los individuos desarrollen sus propias capacidades para desenvolverse adecuadamente en un medio monetizado y logren sus objetivos financieros particulares.

¿Pero está esto realmente ocurriendo? El presente trabajo de investigación busca identificar en qué medida los conocimientos financieros de los colaboradores de la Asociación del Noreste Colombiano, se relacionan con sus capacidades financieras.

\section{Revisión de la literatura}

\section{Educación financiera}

La literatura técnica para ciencias empresariales ha acuñado ante la comunidad científica términos como: alfabetización fi- 
nanciera y cultura financiera o educación financiera, para referirse al proceso de impartir conocimientos financieros que ayuden a las personas a involucrarse en las actividades financieras adecuadamente.

Según la Comisión Nacional para la Protección y Defensa de los Usuarios de Servicios Financieros CONDUCEF (2009), la educación para el siglo XX, tuvo como reto la enseñanza de procesos básicos de alfabetismo; sin embargo, para este nuevo siglo el reto de la educación se centra en la enseñanza del cuidado y formación del patrimonio, es decir, en la denominada educación financiera (citado en Ruiz, 2005); es la misma que debe ser promovida en todos los niveles y estamentos.

Una de las entidades abanderadas a nivel mundial en promover la educación financiera es la Organización para la Cooperación y el Desarrollo Económico OCDE, este importante órgano define la educación financiera como "un proceso que permite la comprensión sobre productos, conceptos y riesgos financieros a través del desarrollo de habilidades sobre riesgos y oportunidades financieras" (Urbiola, 2011).

Este concepto es preciso al indicar que la educación financiera contempla tanto la adquisición de conocimientos (comprensión), como el desarrollo de capacidades (habilidades), para identificar riesgos y aprovechar oportunidades financieras.

En consideración a lo mencionado, DKiyosaki y Lechter (2007), sostienen que una persona que carece de educación financiera no puede ver el interior de una inversión, debido a que el conocimiento financiero permite ver el interior de una inversión y conocer la verdad, los hechos, la ficción, las oportunidades y el riesgo (Pág. 224).

\section{Importancia del conocimiento financiero}

La educación entendida como el proceso destinado a trasmitir conocimientos, valores, habilidades, costumbres y formas de comportarse, o como el "proceso constructivo que al nivel individual se desarrolla desde el nacimiento hasta la edad adulta y se prolonga a nivel social con el desarrollo de la ciencia" (García, 2006), tiene de por sí un componente cognitivo que equivale a la parte teórica del proceso formativo. De allí que, la educación financiera es un área del conocimiento esencial para el manejo de la economía personal, familiar e incluso nacional. (Ruiz, 2005).

Según García (2006), el conocimiento se ha convertido (más de lo que históricamente ha sido), en la base del poder. Por tanto, los conocimientos financieros resultan insustituibles en el proceso de formación; por ello, las personas que tienen conocimientos financieros, los han adquirido mediante el estudio o en su interacción con otras personas, puesto que nadie nace sabiendo contabilidad y finanzas (Amat, 2000).

Asimismo, es necesario que en el proceso de formación; los padres, el gobierno, el sector financiero y la escuela, deben asegurar la transmisión e interiorización en los jóvenes con las bases teóricas claves.

\section{Conocimientos financieros claves}

Resulta poco probable elaborar una lista única y completa de todos los conocimientos financieros útiles; sin embargo, diferentes estudios científicos hacen referencia a algunos que son claves o indispensables. Según el Banco Mundial, los conceptos técnicos evaluados para medir los conocimientos financieros son: Conocimientos matemáticos básicos, manejo 
de terminología financiera, dominio del concepto de interés pagado sobre un préstamo, cálculo de interés simple y cálculo de interés compuesto; conocimientos que son necesarios para tomar decisiones financieras acertadas. (Reddy, Bruhn, y Tan, 2013).

Además, los conocimientos matemáticos básicos juegan un papel fundamental entre los conocimientos financieros, pues sin ellos son difíciles de desarrollar otras competencias. De acuerdo con el Ministerio de Educación Nacional de Colombia y ASOBANCARIA ( 2012), las competencias matemáticas posibilitan "el uso en contexto de los conceptos, proposiciones, teorías y modelos matemáticos representados en el conocimiento y los distintos tipos de pensamiento lógico y matemático imprescindibles y necesarios para desempeñarse en forma activa y crítica en la vida social y política".

Las matemáticas deberían ayudar no tanto a resolver problemas complejos; pues estos son realizados hoy en día por los ordenadores, pero si a tener la capacidad de interpretar, evaluar, discutir y comunicar información matemática y a desarrollar las competencias para resolver los problemas matemáticos de la vida diaria y el trabajo profesional; para lo cual, en muchas ocasiones, incluso, se requieren conocimientos de matemáticas financieras. (Godino, Batanero y Font, 2003)

Ahora bien, el manejo de la terminología financiera es otro conocimiento financiero clave. Es la comprensión del significado de los términos financieros más comunes, lo que le permite al individuo entender la información que recibe. Al respecto, García, Acosta, y Gil (2013), estipulan que el Banco de la Republica de Colombia al examinar las habilidades financieras que tienen los colombianos, plan- tean preguntas referentes a realizar operaciones matemáticas y adicionalmente el dominio de conceptos financieros básicos.

Por su parte, Visco ( 2010), es claro en afirmar que "las familias con más conocimiento acerca de nociones de riesgo y rendimiento, interés compuesto e inflación pueden identificar mejor el abuso y el fraude, así como entender los verdaderos términos de lo que se les ofrece".

De igual forma, el cálculo de interés simple y compuesto es también un conocimiento financiero básico. En el año 2005 la OECD diseñó un programa sobre educación financiera considerando que la educación financiera es un asunto de información y habilidad para comprender las tasas de interés, elaborar presupuestos y comparar préstamos.

Entonces es importante destacar que, la educación financiera es también "una cuestión de comportamiento apropiado, de prudencia, planeación y moderación en el endeudamiento". (Garay, 2013).

\section{Etapas de la formación financiera}

La adquisición de conocimientos y capacidades financieras es un proceso continuo y a largo plazo que debe irse completando al surgir las necesidades en las diferentes etapas de la vida. Además, el éxito de la educación financiera no radica en la acumulación de conocimientos financieros sino en la capacidad de los individuos para usar esa información, de allí que comienza con nociones muy básicas, para pasar luego a nociones más avanzadas relacionadas con el entendimiento de conceptos financieros o el desarrollo de habilidades y actitudes para la gestión de las finanzas personales, las cuales genran cambios positivos en el comportamiento de las personas (García et al., 2013). 
Para un buen programa de educación financiera debe, entre otros, partir de un objetivo muy preciso y realizable; el contenido del material debe ser muy práctico, la utilidad inmediata y accesible inclusive para analfabetos; adicionalmente la forma de enseñar debe ser amena y muy participativa (Gomez Zoto, 2009).

De otra parte, se debe considerar que las necesidades a las que se enfrenta una familia pobre, principalmente de satisfacer necesidades básicas, son diferentes a las de las familias adineradas que temen perder cuando toman decisiones equivocadas (Visco, 2010).

Por esto, es preciso diseñar programas de educación financiera orientados a las necesidades propias de cada segmento de la población.

\section{Capacidades financieras}

En este mismo orden de ideas, la educación financiera no debe limitarse solo a la acumulación de información sobre los productos financieros existentes 0 al deber ser del actuar financiero de los individuos. Por ello, Roa (2010), establece que los individuos pueden no procesar o rechazar la información que tienen a su disposición, o seleccionar aquella que confirma su creencia. Incluso, aunque la haya procesado racionalmente; son capaces de ignorarla y actuar guiados por un impulso espontaneo por lo que creen que es cierto.

En este mismo sentido Urbiola (2011), afirma que "el acceso que los ciudadanos tienen a la información económica y financiera no asegura que puedan utilizarla provechosamente. Es necesario un esquema de educación financiera que busque satisfacer la complejidad de las necesidades individuales".
Estos esquemas de educación deberían estar enfocados a desarrollar en los ciudadanos conocimientos económicos que según (BAC-REDOMATIC, 2008) "sirven para valernos en la vida, comprender la sociedad y mejorar el mundo en que vivimos".

\section{La importancia del conocimiento para el desarrollo de capacidades financieras}

Los conocimientos financieros son esenciales para el desarrollo de capacidades financieras, el ministerio de educación nacional define las competencias como "un saber hacer flexible que puede actualizarse en distintos contextos; es decir, como la capacidad de usar los conocimientos en situaciones distintas de aquellas en la que se aprendieron.

Implica la comprensión del sentido de cada actividad y sus implicaciones éticas, sociales, económicas y políticas"(Ministerio de Educación Nacional de Colombia, 2014). Por tanto, las capacidades financieras son competencias que los individuos desarrollan a partir de los conocimientos financieros que poseen.

Gómez (2009), señala que los conocimientos financieros aplicados, deberían ayudar a mitigar los riesgos de sobreendeudamiento e impulsar la mejor administración de los recursos en el hogar.

Asimismo, contempla que para la organización Microfinance Opportunities, el trasmitir conocimientos, habilidades y actitudes mediante la educación financiera, es necesario para que la gente pueda adoptar buenas prácticas de manejo del dinero, siendo que permite la generación de ingresos, manejar los gastos, tener ahorros y manejar el endeudamiento e inversión. 
Otro elemento que debe tomarse en cuenta, es que sin los conocimientos necesarios es muy difícil para las personas desarrollar las capacidades financieras, puesto que es necesario el conocimiento para generar una actitud traducida en capacidad, por ello, ya en las escrituras bíblicas Pablo escribió a los romanos "transformaos mediante la renovación de vuestro entendimiento" (Romanos 12:2 Versión Reina - Valera 1960).

Por tanto, el cambio debe ocurrir primero en la mente de las personas y para que el cambio sea duradero, deben comprender claramente porqué hacen las cosas y qué beneficio obtienen con ello.

En este sentido, Meraz y García (2008), estipulan que es necesario "favorecer la capacidad de las personas para tomar decisiones adecuadas, a través de la información, el razonamiento, la voluntad y responsabilidad de sus elecciones".

Por su parte, Gomez Zoto (2009), quien analizó los programas de educación financiera de más de una decena de países, encontró que el impacto de los programas de educación financiera, en los cuales se imparten conocimientos, tienden a ser pequeños; pues la capacidad de ahorro depende de los hábitos de consumo que traen las personas, y que el impacto es mayor en los días siguientes a la finalización del curso y disminuye con los días, por lo que es necesario programas de reinducción para que las personas logren anclar lo aprendido y en lo posible lo pongan en práctica en el corto plazo.

\section{Capacidades financieras claves}

La Organización para la Cooperación y el Desarrollo Económico, en relación a las buenas prácticas para la educación y con- cientización financiera, recomendó promover el fortalecimiento de la capacidad financiera basado en información e instrucción financiera apropiada, tomando medidas pertinentes cuando se observan deficiencias en las capacidades financieras esenciales. (Principles, 2005)

Es así como, una de las capacidades financieras claves o esenciales es el ahorro. Algunos autores como Gomez Zoto (2009), afirman que a las personas que no tienen el hábito de ahorrar se les dificulta desarrollar otras capacidades financieras.

Según Iregui, Melo, Ramire, y Tribín (2016), una de las razones por las que el ahorro es tan importante, es porque ofrece seguridad financiera para el periodo de retiro o jubilación, sirve para financiar gastos de vivienda, salud, educación y ayuda a amortiguar eventos inesperados como enfermedades, pérdidas de empleos y malas cosechas; también sirve como fuente de recursos para establecer un negocio. Adicionalmente el ahorro fortalece el dominio propio del individuo en relación al consumo y al uso del dinero.

Por ello, para que exista el ahorro es necesario que el estado de operaciones o estado de resultados de una persona sea positivo, y como siempre existe la posibilidad de gastar más, es útil apoyarse en un presupuesto.

Hacer un presupuesto y ajustarse a este es otra de las capacidades financieras que deberían desarrollar los individuos. Según el BAC-REDOMATIC (2008), es importante que exista prudencia en las compras, con el fin de no solo obtener por el dinero un valor equitativo, sino que además sirva para hacer que lo comprado sirva al máximo; de manera tal que permita equilibrar el presupuesto; y es 
más, aun poder economizar algo; sin embargo su consideración son tareas que exigen habilidad administrativa.

Cabe precisar que aparte de constituirse en mapa financiero para llegar al destino deseado, el presupuesto, permite disciplinarse en el momento a fin de tener un bienestar futuro. De allí que, mediante "un plan bien orientado es posible gastar de manera inteligente, contribuyendo al logro de las metas a corto, mediano y largo plazo" (Hiciano, 2014).

Además, otra de las capacidades financieras claves es la orientación al logro de los objetivos financieros, esto implica prever y diseñar un horizonte económico deseado mediante la planificación.

Al respecto, el Ministerio de Educación Nacional de Colombia y ASOBANCARIA (2012), estipulan que planear es proyectarse en el futuro, para ello es necesario tener la capacidad de fijar metas en el tiempo y definir acciones y estrategias; es decir, determinar la manera como poder cumplirlas.

Finalmente, por su parte Meraz, García, y Loreto (2008), determinan que en la mayoría de la población no existe la visión de mediano y largo plazo en lo que concierne a sus finanzas, por ello recomiendan promover entre la población y los hogares una cultura de anticipación y planeación que permita construir mejores condiciones de vida para el presente y el futuro.

Las capacidades financieras y la calidad de vida.

Cejudo (2007), define las capacidades como oportunidades para llevar una u otra clase de vida y es eso precisamente en lo que consisten las capacidades financieras, en oportunidades para planear la forma como se generará y usará los recursos, la parte que se conservará y la manera en que ellos contribuirán el bienestar en el futuro.

Quiere decir entonces, que las capacidades están ampliamente relacionadas con la influencia y el poder, incluso poder sobre otros. Entendiéndose la capacidad como libertad para actuar de determinada manera, esa misma libertad inviste a su poseedor de capacidad y poder para lograr un objetivo propuesto.

Mirándolo desde este punto de vista, cabe afirmar que las capacidades financieras le dan poder a una persona incluso sobre otra en la medida en que ésta pueda pagar por las cosas que desea y haya otras personas que estén dispuestas a ceder un bien o someter su voluntad a otro a cambio de un recurso.

Al respecto, Jaramillo y Daher (2015), han mostrado como producto de su labor investigativa, los efectos del ahorro en el aumento del ingreso de las familias; esto es de entenderse, pues teóricamente a mayor ahorro, mayor posibilidad de adquirir bienes de capital, y a mayor capital, mayor ingreso.

De allí que, los autores sostienen que contar con dinero permite financiar proyectos y adquirir bienes propios; lo cual tiene consecuencias positivas sobre la valoración personal, la autoestima, la satisfacción y el orgullo. Por tanto, "el bienestar financiero guarda una estrecha relación con el bienestar personal, familiar y social, y éste refleja el progreso de una nación" (Meraz y García, 2008). 
Consecuencias de las escasas capacidades financieras.

A la luz de lo que dijera White (1890), las consecuencias de las escasas capacidades financieras, es que muchas familias pobres son pobres porque gastan su dinero tan pronto como lo reciben.

En consecuencia, tal actitud lo que muestra es la ausencia de presupuesto, ahorro y de orientación al logro de objetivos futuros; inevitablemente este comportamiento conlleva a la pobreza.

Asimismo, Trump y Kiyosaki (2007), consideran que muchos de los problemas financieros actuales existen porque no se resolvieron cuando surgieron; enfatizando que en vez de incrementar la inteligencia financiera de la población, se le enseña a esperar que el gobierno resuelva sus problemas y la situación de pobreza continúa asechando a los menos instruidos.

En conclusión, entre los altos costos de las decisiones financieras miopes a los que se refiere Visco (2010), se pueden enumerar: proyectos inconclusos (Lucas 14:30 Versión Reina - Valera 1960), discusiones y desintegración familiar (Rodríguez, 2015), sueños frustrados, falta de liquidez, sobreendeudamiento, pobreza, desánimo y desmotivación (White, 1890), esclavitud (Proverbios 22:7 Versión Reina - Valera 1960), estrés y problemas mentales (Mansilla, Denegri \& Álvarez, 2016).

Finalmente, Ashraf, Trivelli, Yancari, y Zapata afirman que, "disponer de dinero extra para financiar proyectos personales y adquirir bienes propios tiene efectos positivos sobre la valoración personal y la autonomía", (citado en Jaramillo y Daher, 2015).

\section{Métodos}

Esta investigación corresponde a un estudio cuantitativo descriptivo y correlacional no experimental, de corte trasversal para medir la relación entre los conocimientos financiero de los colaboradores de la Iglesia Adventista Asociación del Noreste Colombiano y sus capacidades financieras. Para estimar el grado de relación se utilizó el coeficiente de correlación de Pearson.

La población objeto de estudio estuvo conformada por los 150 empleados de la Iglesia Adventista del Séptimo día de Colombia Sección Asociación del Noreste Colombiano, todos adultos mayores de 18 años, de los cuales 19 son administradores de campo o de colegios, 28 son pastores de distrito, 9 son de servicios generales, 15 son empleados de oficina y 79 son maestros de escuelas y colegios.

De la población antes mencionada se tomó una muestra probabilística de 114 personas.

Para la recolección de datos se utilizó el instrumento (cuestionario) de medición con el empleo de la técnica de encuesta, basada en la escala mixta LikertThuerstone.

El instrumento aplicado con alta confiabilidad ( $\alpha=0.877$ ), está compuesto por 65 ítems, de los cuales 33 están direccionados a medir la variable conocimientos financieros, 28 ítems la variable capacidades financieras y 4 ítems que ofrecen información al investigador sobre la manera que en los encuestados usan el dinero que les sobra y el ingreso ocasional que reciben, tal como se verifica en la Tabla 1. 
Tabla 1

Estructura y confiabilidad del instrumento de conocimientos y capacidades financieras

\begin{tabular}{lcc}
\hline Dimensión & $\begin{array}{c}\text { Número de } \\
\text { Ítems }\end{array}$ & Alfa de Cronbach \\
\hline Conocimientos matemáticos básicos & 10 & \\
Manejo de terminología financiera & 13 & \\
Cálculo de interés simple y compuesto aplicado al préstamo y a la & 10 & 0,759 \\
inversión & & \\
\hline Conocimientos Financieros & 33 & \\
\hline Planificación del presupuesto & 10 & \\
Capacidad de ahorro & 3 & \\
Orientación al logro de los objetivos & 15 & 0,869 \\
\hline Capacidades Financieras & 28 & \\
\hline
\end{tabular}

\section{Resultados}

\section{Análisis descriptivo}

De acuerdo con sus características sociodemográficas, la población encuestada según la Tabla 2, en su mayoría tiene un estado civil casado/unión libre $(62,7 \%)$, un tercio de la población aproximadamente es soltero $(33,6)$; en relación con el nivel de estudios en su gran mayoría tiene formación universitaria profesional $(75,5 \%)$, y un significativo $11,8 \%$ cuenta con estudios de postgrado.

En cuanto a la edad casi la mitad está entre los 25 a 34 años de edad, el otro $40 \%$ está entre los 35 y 54 años, que es la edad más productiva. Referente al género la población es casi homogénea, prevaleciendo el género femenino $(51,8 \%)$, respecto al $48,2 \%$ de género masculino. Observando los resultados demográficamente, se encontró que los hombres mostraron mayor conocimiento y capaci- dad financieras mayores de 55 años los que mostraron tener mayores conocimientos financieros y mayor capacidad de planear el presupuesto, pero fueron superados ligeramente por los empleados con edades entre 25 y 34 años en cuanto a la orientación al logro de los objetivos y la capacidad de ahorro.

Los empleados casados apuntaron a una mayor planificación del presupuesto y los separados o divorciados los que mostraron tener una mayor orientación al logro de los objetivos financieros, pero una menor capacidad de ahorro.

Finalmente, analizando los datos por nivel educativo, los empleados con posgrado superaron a los universitarios en cuanto a sus conocimientos financieros, pero fueron los empleados con nivel académico universitario los que obtuvieron mejores resultados en cuanto a las capacidades financieras.

Tabla 2

Datos sociodemográficos de la población de estudio

\begin{tabular}{llllllll}
\hline \multicolumn{2}{c}{ Estado Civil } & \multicolumn{2}{c}{ Nivel de Estudios } & \multicolumn{2}{c}{ Edad } & & Sexo \\
\hline Soltero & $33,6 \%$ & Sin Nivel Educativo & $0,9 \%$ & De 18 a 24 años & $12,7 \%$ & Femenino & $51,8 \%$ \\
& & & & & & & \\
Casado/Uni & $62,7 \%$ & Primaria & $0,9 \%$ & De 25 a 34 años & $41,8 \%$ & Masculino & $48,2 \%$ \\
ón & & & & & & &
\end{tabular}




\begin{tabular}{|c|c|c|c|c|c|}
\hline $\begin{array}{l}\text { Separado/ } \\
\text { Divorciado }\end{array}$ & $3,6 \%$ & Bachillerato & $2,7 \%$ & De 35 a 44 años & $21,8 \%$ \\
\hline \multirow[t]{3}{*}{ Viudo } & $0 \%$ & Técnica superior & $8,2 \%$ & De 45 a 54 años & $18,2 \%$ \\
\hline & & Universitaria & $75,5 \%$ & Más de 55 años & $5,5 \%$ \\
\hline & & Posgrado & $11,8 \%$ & & \\
\hline
\end{tabular}

Además, al comparar los resultados descriptivos obtenidos sobre las capacidades financieras, con los resultados del estudio del Banco Mundial, según la Tabla 3 , se encontraron resultados similares y diferencias estadísticamente significativas, la más resaltante es respecto a la capacidad de contar con estrategias para asegurar la provisión de recursos suficientes para la educación universitaria de sus hijos; en el cual sólo el $35 \%$ de los colaboradores de la población de estudio manifestó tener una estrategia para asegurar la provisión de recursos suficientes; en relación con otro estudio, en el cual más del $85 \%$ de los encuestados informan que tie- ne planes para costear la educación de sus hijos.

Otro dato significativo es el que hace referencia al endeudamiento en el cual se determinó que existe una tasa de endeudamiento muy superior entre los colaboradores de la Asociación del noreste (91\%), con respecto al promedio de los colombianos $(28 \%)$; por otra parte, se determinó que los colaboradores de la iglesia adventista del noreste colombiano al tener un ingreso fijo hacen menos provisión para gastos imprevistos en comparación con el promedio de los colombianos.

Tabla 3

Comparativos de los resultados del estudio, con resultados de la encuesta realizada por el Banco

\begin{tabular}{|c|c|c|}
\hline $\begin{array}{c}\text { Encuesta sobre Conocimientos y } \\
\text { capacidades financieras aplicada a los } \\
\text { empleados de la Asociación del Noreste } \\
\text { Colombiano }\end{array}$ & $\begin{array}{c}\text { Resultados de la encuesta sobre } \\
\text { capacidades financieras en Colombia } \\
\text { realizada por el Banco Internacional de } \\
\text { Reconstrucción y fomento/Banco } \\
\text { Mundial }\end{array}$ & OBSERVACIONES \\
\hline $\begin{array}{l}\text { El } 48 \% \text { tienen un plan para cubrir los gastos de } \\
\text { su hogar en la vejez }\end{array}$ & $\begin{array}{l}\text { El } 41 \% \text { tienen planes para pagar por completo } \\
\text { los gastos de su vejez }\end{array}$ & $\begin{array}{l}\text { Los empleados de la Asociación del Noreste tienen } \\
\text { mayores posibilidades de cubrir sus gastos en la } \\
\text { vejez que el promedio de los colombianos. }\end{array}$ \\
\hline $\begin{array}{l}1 \text { de cada } 10 \text { encuestados está totalmente de } \\
\text { acuerdo en que podría cubrir un gasto } \\
\text { importante imprevisto }\end{array}$ & $\begin{array}{l}1 \text { de cada } 5 \text { colombianos manifestó poder } \\
\text { afrontar gastos importantes imprevistos }\end{array}$ & $\begin{array}{l}\text { Esta escala es desventajosa para los empleados de } \\
\text { la iglesia adventista del noreste colombiano. } \\
\text { Podría deberse a que al tener un ingreso fijo } \\
\text { hacen menos provisión para gastos imprevistos. }\end{array}$ \\
\hline $\begin{array}{l}\text { Al } 33 \% \text { de los empleados se les dificulta calcular } \\
\text { una tasa de interés simple. }\end{array}$ & $\begin{array}{l}\text { El } 81 \% \text { de la población no es capaz de calcular } \\
\text { una tasa de interés simple }\end{array}$ & $\begin{array}{l}\text { En este sentido, los empleados de la iglesia } \\
\text { adventista obtuvieron resultados mejores que el } \\
\text { promedio de los colombianos, esto podría } \\
\text { deberse al alto nivel de alfabetización que hay } \\
\text { entre los primeros }\end{array}$ \\
\hline $\begin{array}{l}\text { El 91\% de los encuestados reconoció tener algún } \\
\text { tipo de deuda. }\end{array}$ & $\begin{array}{l}\text { El } 28 \% \text { de los encuestados manifestó que } \\
\text { debía dinero a alguien }\end{array}$ & $\begin{array}{l}\text { Existe una tasa de endeudamiento muy superior } \\
\text { entre los empleados de la Asociación del noreste } \\
\text { que entre el promedio de los colombianos }\end{array}$ \\
\hline $\begin{array}{l}\text { El } 88 \% \text { de los encuestados pudo responder } \\
\text { correctamente por lo menos } 3 \text { de cada } 5 \text { (o } 22 \\
\text { de las } 33 \text { ) preguntas relacionadas con los } \\
\text { conocimientos financieros }\end{array}$ & $\begin{array}{l}\text { Más del } 60 \% \text { de los colombianos respondió } \\
\text { correctamente al menos tres de las cinco } \\
\text { preguntas sobre conocimientos financieros }\end{array}$ & $\begin{array}{l}\text { Este indicador muestra un mayor grado de } \\
\text { conocimiento financiero entre los empleados de la } \\
\text { Asociación del Noreste }\end{array}$ \\
\hline $\begin{array}{l}\text { El } 57 \% \text { indicó que hacía un plan de sus gastos } \\
\text { bastante exacto, mientras que el } 22 \% \text { manifestó } \\
\text { que no hace presupuesto. }\end{array}$ & $\begin{array}{l}\text { El } 45 \% \text { dijo que hacía un presupuesto exacto, } \\
50 \% \text { planes aproximados y } 6 \% \text { ningún plan }\end{array}$ & $\begin{array}{l}\text { Este indicador muestra una mayor tendencia } \\
\text { entre los empleados de la Asociación del noreste a } \\
\text { hacer un plan exacto. }\end{array}$ \\
\hline
\end{tabular}




\begin{tabular}{|c|c|c|}
\hline $\begin{array}{l}\text { Solo el } 35 \% \text { de los empleados manifestó tener } \\
\text { una estrategia para asegurar la provisión de } \\
\text { recursos suficientes para la educación } \\
\text { universitaria de sus hijos. }\end{array}$ & $\begin{array}{l}\text { Más del } 85 \% \text { de los encuestados informo que } \\
\text { tiene planes para costear la educación de sus } \\
\text { hijos }\end{array}$ & Este indicador es significativamente diferente. \\
\hline $\begin{array}{l}\text { El } 50 \% \text { de los empleados mayores de } 55 \text { años } \\
\text { está ejecutando algún plan para cubrir los gastos } \\
\text { de su hogar en la vejez }\end{array}$ & $\begin{array}{l}\text { Solo el } 41 \% \text { de la población de menos de } 60 \\
\text { años ha tomado previsión para poder pagar la } \\
\text { totalidad de sus gastos durante la vejez }\end{array}$ & $\begin{array}{l}\text { Este indicador muestra que solo cerca del } 50 \% \text { de } \\
\text { ambas poblaciones, tienen planes o está haciendo } \\
\text { provisiones para cubrir sus gastos en la vejez }\end{array}$ \\
\hline $\begin{array}{l}\text { El } 75 \% \text { de los encuestados mostró tener un } \\
\text { fuerte compromiso con el logro de sus objetivos } \\
\text { financieros. }\end{array}$ & $\begin{array}{l}\text { El } 80 \% \text { de los encuestados expresó una fuerte } \\
\text { orientación al logro de los objetivos } \\
\text { financieros. }\end{array}$ & Resultados similares \\
\hline $\begin{array}{l}\text { El } 54 \% \text { de los empleados manifestó que en los } \\
\text { últimos } 12 \text { meses ha ahorrado algún monto. El } \\
22 \% \text { ha ahorrado por lo menos el } 6 \% \text { de su } \\
\text { ingreso mensual. }\end{array}$ & $\begin{array}{l}\text { La mayor parte de la población no ahorra, } \\
\text { quienes lo hacen, ahorran por lo general para } \\
\text { situaciones imprevistas. }\end{array}$ & $\begin{array}{l}\text { Existe una mayor capacidad de ahorro entre los } \\
\text { empleados de la Asociación del Noreste que entre } \\
\text { el colombiano promedio. }\end{array}$ \\
\hline $\begin{array}{l}\text { El } 66 \% \text { de los encuestados mostró tener facilidad } \\
\text { para realizar operaciones matemáticas básicas, } \\
\text { el } 60 \% \text { pudo identificar un porcentaje mal } \\
\text { calculado. El } 58 \% \text { respondió correctamente las } \\
\text { preguntas relacionadas con la definición de } \\
\text { inflación. }\end{array}$ & $\begin{array}{l}\text { La mayoría de los encuestados respondió } \\
\text { correctamente las preguntas sobre } \\
\text { matemáticas básicas, la definición de } \\
\text { inflación y el concepto de interés de un } \\
\text { préstamo }\end{array}$ & $\begin{array}{l}\text { Resultados similares, ligeramente mejores entre } \\
\text { los empleados de la Asociación del Noreste }\end{array}$ \\
\hline $\begin{array}{l}\text { Respuestas correctas sobre } \\
\text { Valor temporal del dinero } 63 \% \\
\text { Cálculo de interés simple } 54 \% \\
\end{array}$ & $\begin{array}{l}\text { Respuestas correctas sobre Valor temporal } \\
\text { del dinero } 69 \% \\
\text { Cálculo de interés simple } 35 \% \\
\end{array}$ & $\begin{array}{l}\text { Los empleados de la Asociación del Noreste } \\
\text { mostraron mayor facilidad para hacer cálculos de } \\
\text { interés simples }\end{array}$ \\
\hline $\begin{array}{l}\text { Los hombres y los empleados con posgrados } \\
\text { obtuvieron mejores resultados en las preguntas } \\
\text { relacionadas con los conocimientos financieros }\end{array}$ & $\begin{array}{l}\text { Los hombres y quienes tenían una educación } \\
\text { superior y un ingreso más alto tenían más } \\
\text { probabilidades de responder correctamente } \\
\text { las preguntas sobre conocimientos financiero }\end{array}$ & Resultados similares \\
\hline $\begin{array}{l}\text { El estudio mostró que existe una relación } \\
\text { estadísticamente significativa del } 44 \% \text { entre los } \\
\text { conocimientos financieros y las capacidades } \\
\text { financieras de los empleados de la Asociación } \\
\text { del Noreste colombiano de los Adventistas del } \\
\text { Séptimo día }\end{array}$ & $\begin{array}{l}\text { En Colombia, una mayor puntuación en } \\
\text { conocimientos financieros se asocia a una } \\
\text { mayor medida de capacidades financieras de } \\
5 \text { a } 10 \text { componentes de esta última }\end{array}$ & Resultados similares \\
\hline $\begin{array}{l}\text { Se encontró que los conocimientos financieros } \\
\text { de los empleados se correlacionan con su } \\
\text { capacidad de ahorro, su orientación al logro de } \\
\text { los objetivos y la planificación del presupuesto. }\end{array}$ & $\begin{array}{l}\text { La encuesta encontró que los conocimientos } \\
\text { financieros guardan una estrecha relación } \\
\text { con seguimiento a los gastos, orientación al } \\
\text { logro de los objetivos y comportamiento no } \\
\text { impulsivo }\end{array}$ & Resultados similares \\
\hline
\end{tabular}

*Fuente: Encuesta nacional sobre capacidades financieras en Colombia, realizada por el Banco Internacional de Reconstrucción y Fomento (Reddy, Bruhn, \& Tan, 2013).

En cuanto a los hábitos de ahorro, endeudamiento y uso de excedentes, en la Tabla 4 relacionada con el endeudamiento, se encontró que solo el $7,3 \%$ de los empleados no ha tenido deudas en los últimos 12 meses, y que la deuda del
$26,4 \%$ de la población aumentó en este mismo periodo de tiempo. Estos resultados dejan entrever un alto nivel de endeudamiento entre los encuestados, lo que les representa un gasto elevado en intereses.

Tabla 4

Estado de obligación de deuda último año

\begin{tabular}{lrr}
\hline Durante los últimos 12 meses el estado de su obligación (deuda) ha: & F & $10,9 \%$ \\
\hline Aumentado significativamente & 12 & $15,5 \%$ \\
Aumentado levemente & 17 & $27,3 \%$ \\
Se ha mantenido estable & 30 & $19,1 \%$ \\
Disminuido un poco & 21 & $20,0 \%$ \\
Disminuido significativamente & 22 & $7,3 \%$ \\
No he tenido deudas en los últimos 12 meses & 8 & \\
\hline
\end{tabular}

En relación con el ahorro, en la Tabla 5 se verifica que solo el $31.8 \%$ ajustó los gastos a su ingreso y el $54.5 \%$ ahorró mensualmente; sin embargo, la mayoría de quienes ahorraron, solo ahorró entre el $1 \%$ y el $5 \%$ de su ingreso. Estos resultados dejan entrever la escasa planificación y proyección de gastos futuros. 
Tabla 5

Ahorro durante los últimos 12 meses

\begin{tabular}{lcc}
\hline ¿En los últimos 12 meses ha ahorrado algún porcentaje de su ingreso mensual? & $\mathrm{N}$ & $\%$ \\
\hline No, por el contrario, me he endeudado & 15 & 13,6 \\
No, he ajustado mi gasto al ingreso & 35 & 31,8 \\
Entre el 1\% y el 5\% de mi ingreso & 35 & 1,8 \\
Entre el 6\% y el 10\% de mi ingreso & 16 & 9,5 \\
Más del 10\% de mi ingreso & 9,2 \\
\hline
\end{tabular}

Además, cuando se le preguntó a los encuestados cuánto tiempo podría cubrir sus gastos sin pedir dinero prestado si perdiera su principal fuente de ingreso, el $38,2 \%$ manifestó poder cubrir sus gastos por lo menos un mes, pero menos de tres meses; el $34,5 \%$ dijo poder cubrir sus gastos tan solo un mes, y solo el $12,7 \%$ indicó poder cubrir sus gastos más de seis me- ses sin pedir dinero prestado. Se puede deducir que los ahorros de $72,7 \%$ de los empleados son inferiores a tres veces su ingreso mensual, lo que los hace vulnerables al endeudamiento ante la pérdida de su empleo y limita sus posibilidades de aprovechar oportunidades de inversión, tal como se verifica en la Tabla 6.

Tabla 6

Relación Fondos de Ahorro Disponibles

\begin{tabular}{lcc}
\hline En caso de que usted pierda su principal fuente de ingreso, ¿cuánto tiempo & $\mathrm{n}$ \\
podría seguir cubriendo sus gastos sin pedir prestado dinero? & 13 & 11,8 \\
\hline Menos de una semana & 25 & 22,7 \\
Por lo menos una semana, pero menos de un mes & 42 & 16,2 \\
Por lo menos un mes, pero menos de tres meses & 14,5 \\
Por lo menos tres meses, pero menos de 6 meses & 14 & 12,7 \\
Más de seis meses & 16 \\
\hline
\end{tabular}

\section{Análisis inferencial}

Bajo el análisis inferencial, al determinar la relación entre conocimientos financieros y planificación del presupuesto, en la Tabla 7 según el nivel de significancia de $0,001(p<0,05)$, existe una correlación positiva baja o débil, teniendo en cuenta la valoración del rho de Spearman $(r=$ $0,301)$; lo que significa que cuanto más conocimiento financiero posean los empleados, tendrán una mayor propensión a planificar su presupuesto y a ajustarse a él. Pese a que técnicamente la correlación entre los conocimientos financieros y la planificación del presupuesto es débil, en la práctica una incidencia del $30.1 \%$ en este sentido, es de gran relevancia por la importancia que tiene el presupuesto para lograr unas finanzas saludables.

Tabla 7

Relación entre conocimientos financieros y planificación del presupuesto

\begin{tabular}{lccc}
\hline & \multicolumn{3}{c}{ Conocimientos financieros } \\
\cline { 2 - 4 } & Rho de Spearman & $\mathrm{p}$ valor & $\mathrm{N}$ \\
\hline Planificación del presupuesto &, $301^{* *}$ &, 001 & 110 \\
\hline
\end{tabular}

${ }^{* *}$ Fuerza: $\pm 0.0, \pm 0.09$ NULA; $\pm 0.1, \pm 0.39$ DEBIL; $\pm 0.4, \pm 0.69$ MODERADA; $\pm 0.7, \pm 0.95$ FUERTE; $\pm 0.96, \pm 1.0$ PERFECTA) 
En cuanto a los conocimientos financieros y su relación con la capacidad de ahorro, de acuerdo con la Tabla 8 entre la variable conocimientos financieros y la dimensión capacidad de ahorro existe una correlación positiva baja o débil según lo indica la rho de Spearman que correspon- de a 0.294 y un nivel de significancia de $0.002(p<0.05)$, esto significa que cuanto mayor se conozca sobre finanzas, mayor será la capacidad de ahorro, o de lo contrario, cuanto menos conozca el empleado sobre finanzas, menor será su capacidad o disposición a ahorrar.

Tabla 8

Relación de conocimientos financieros y capacidad de ahorro

\begin{tabular}{lccc}
\hline & \multicolumn{3}{c}{ Conocimientos financieros } \\
\cline { 2 - 4 } & Rho de Spearman & p valor & $\mathrm{N}$ \\
\hline Capacidad de ahorro &, $294^{* *}$ &, 002 & 110 \\
\hline
\end{tabular}

** Fuerza: $\pm 0.0, \pm 0.09$ NULA; $\pm 0.1, \pm 0.39$ DEBIL; $\pm 0.4, \pm 0.69$ MODERADA; $\pm 0.7, \pm 0.95$ FUERTE; $\pm 0.96, \pm 1.0$ PERFECTA)

En relación con los conocimientos financieros y la orientación al logro de los objetivos, en la Tabla 9 se evidencia que entre la variable conocimientos financieros y la dimensión orientación al logro de los objetivos existe una correlación positiva media o moderada, según lo indica el $r$ de Pearson que corresponde a 0.457 y un nivel de significancia de 0.000 $(p<0.05)$. Esto significa, que cuanto ma- yor se conozca sobre finanzas, mayor será la orientación al logro de los objetivos financieros; reciba capacitación financiera y aumenten sus conocimientos, tendrá una mayor orientación a fijarse objetivos financieros y a alinear sus acciones para lograrlos; adicionalmente le permitirá prever con mayor facilidad el futuro y se le facilitará tomar acciones para enfrentarlo.

Tabla 9

Relación entre conocimientos financieros y orientación al logro de los objetivos financieros

\begin{tabular}{lccc}
\hline & \multicolumn{3}{c}{ Conocimientos financieros } \\
\cline { 2 - 4 } & Rho de Spearman & $p$ valor & $\mathrm{N}$ \\
\hline Orientación al logro de los objetivos financieros &, $457^{* *}$ &, 000 & 110 \\
\hline${ }^{* *}$ Fuerza: $\pm 0.0, \pm 0.09$ NULA $\pm 0.1, \pm 0.39$ DEBIL; $\pm 0.4, \pm 0.69$ MODERADA; $\pm 0.7, \pm 0.95$ FUERTE; $\pm 0.96, \pm 1.0$ PERFECTA)
\end{tabular}

Finalmente, en relación con las capacidades financieras y conocimientos financieros, tal como se aprecia en la Tabla 10, entre las variables existe una correlación positiva estadísticamente significativa, según lo indica el $r$ de Pearson que corresponde a 0.442 y un nivel de significancia de $0.000(p<0.05)$; es decir, que sí existe una relación entre los conocimientos financieros y las capacidades financieras de los colaboradores de la Iglesia Adventista del Séptimo día Sección Asociación del Noreste Colombiano.

Por tanto, en la medida en que los conocimientos sobre cálculo de operaciones matemáticas básicas, terminología financiera y cálculo de interés simple y compuesto aumentan, también aumenta su capacidad para planificar su presupuesto, 
financieros.

Tabla 10

Relación entre las Variables capacidades financieras y conocimiento financiero

\begin{tabular}{lccc}
\hline & \multicolumn{3}{c}{ Conocimientos financieros } \\
\cline { 2 - 4 } Capacidades financieras & Rho de Spearman & $p$ valor & $\mathrm{N}$ \\
\hline${ }^{* *}$ Fuerza: $\pm 0.0, \pm 0.09$ NULA; $\pm 0.1, \pm 0.39$ DEBIL; $\pm 0.4, \pm 0.69$ MODERADA; $\pm 0.7, \pm 0.95$ FUERTE; $\pm 0.96, \pm 1.0$ PERFECTA) \\
\hline
\end{tabular}

\section{Conclusiones}

Existe una relación estadísticamente significativa positiva del $47 \%(0.476)$, entre los conocimientos financieros de los colaboradores de la Iglesia Adventista del Noreste Colombiano y sus capacidades financieras.

Lo que significa que, los conocimientos financieros adquiridos redundarán significativamente en mejores capacidades para desenvolverse financieramente. El factor más débil en la población objeto de estudio fueron los conocimientos financieros y sus conceptos claves, por lo que se debe argumentar que un proceso de formación y reeducación financiera es de suma importancia para anclar los conocimientos financieros con las capacidades, las cuales mostraron un nivel mayor de presencia en la población.

Siendo que la planificación del presupuesto de los colaboradores de la Asociación del Noreste Colombiano depende por lo menos en un $30,1 \%$ de sus conocimientos financieros; pese a que la relación es baja, es relevante por la importancia que tiene el presupuesto para la salud financiera de las personas.

Por otra parte, considerando que una de las capacidades financieras más importantes y difíciles de desarrollar es la capacidad de ahorro; cabe recalcar que los conocimientos financieros juegan un papel de suma importancia, de allí que, el estudio muestra que tienen una relación directa positiva del $29,4 \%$ en la capacidad de ahorro. Asimismo, tienen una relación media o moderada del $45,7 \%$ con la orientación al logro de los objetivos, es decir, tener este tipo de conocimientos ayuda a visualizar un objetivo deseado y a alinear sus decisiones para lograrlo.

Finalmente, los resultados encontrados al igual que otros estudios en cuanto a la medición de capacidades financieras en Colombia, como Reddy et al. (2013) y Mejía (2015), determinan que las mujeres tienen menor capacidad de ahorro y menor orientación al logro de los objetivos financieros que los varones.

De allí que, uno de los mayores retos a los que se enfrenta la organización adventista de la asociación del noreste de Colombia, en materia de educación financiera, es el de nivelar los conocimientos financieros de los empleados del sexo femenino con los de los empleados varones; dado que estas obtuvieron menor números de aciertos que los hombres en las preguntas relacionadas con las tres dimensiones de los conocimientos financieros. 


\section{Referencias}

Amat, O. (2000). Contabilidad y finanzas para no financieros. Curso de gestión empresarial.

BAC-REDOMATIC. (2008). Libro Maestro de Educación Financiera, Un Sistema para Vivir Mejor. Innova Technology, S.A (Vol. 1). http://doi.org/10.1017/CBO9781107415 324.004

Cejudo Córdoba, R. (2007). Capacidades y libertad. Una aproximación a la teoría de Amartya Sen. Revista Internacional de Sociologia RIS, LXV, N ${ }^{\circ}$ 47(MayoAgosto), 9-22.

Esguerra, M. del P. (2011). Medición y evaluación de la educación económica y financiera en Colombia: la experiencia del Banco de la República, 28. Retrieved from

https://www.fogafin.gov.co/web/imagene s/file/Noticias/II TALLER EEF/4_ Maria del Pilar Esguerra -Encuesta de carga y EF.pdf

Garay, G. (2013). Índice de alfabetismo financiero, la cultura y la educación financiera.

García, N., Acosta P, F. A., \& R, Gil, J. L. (2013). Determinantes de la Alfabetización Financiera de la Población Bogotana Bancarizada.

García, N., Grifoni, A., \& López, J. (2013). La educación financiera en América Latina y el Caribe. Serie Políticas Públicas y Transformación Productiva N ${ }^{\circ} 12$ CAF. Retrieved from http://www.oecd.org/daf/fin/financialeducation/OECD_CAF_Financial_Educ ation_Latin_AmericaES.. .pdf

García, R. (2006). Epistemología y Teoría del Conocimiento. Salud Colectiva.
Godino, J. D., Batanero, C., \& Font, V. (2003). Fundamentos de la enseñanza y el aprendizaje de las matemáticas para maestros. Retrieved from http://www.ugr.es/ jgodino/edumatmaestros/manual/1_Fundamentos.pdf

Gomez Zoto, F. (2009). Educacion Financiera: Retos y Lecciones a Partir de Experiencias Representativas del Mundo. Bogotá. Retrieved from https://es.scribd.com/document/537512 33/educacion-financiera

Hiciano, W. (2014). Hablemos de dinero (1st ed.).

Iregui, Ana; Melo, Llgia; Ramirez, Maria; Tribín, A. (2016). Ahorro de los hogares de ingresos medios y bajos de las zonas urbana y rural en Colombia.

Jaramillo, A., \& Daher, M. (2015). El ahorro como estrategia de intervención social para la superación de la pobreza: Estudio cualitativo sobre experiencias de ahorro de personas Chilenas. Universitas Psychologica, 14(4), 12691284.

http://doi.org/10.11144/Javeriana.up144.aeis

Kiyosaki, R., \& Lechter, S. (2007). Guía Para Invertir En qué invierten los ricos ja diferencia de las clases media y pobre!

Mansilla, L., Denegri, M., \& Álvarez, B. (2016). Relación entre actitudes hacia el endeudamiento y locus de control del consumidor en estudiantes universitarios. Suma Psicológica, 23(1), 1-9.

http://doi.org/10.1016/j.sumpsi.2015.11. 002

Mejía, D. (2015). Resultados de las encuestas de medición de capacidades financieras en los países andinos Bolivia, Colombia, Ecuador y Perú. Banco de Desarrollo de América Latina, 24-63. 
Meraz Rios, P. (2008). Primera Encuesta Sobre Cultura Financiera en México, 36. Retrieved from https://gruporepensares.files.wordpress. com/2011/11/cfmexico.pdf

Ministerio de Educación Nacional de Colombia. (2014). Foro Educativo Nacional 2014: Ciudadanos Matemáticamente Competentes.

Ministerio de Educación Nacional de Colombia, \& ASOBANCARIA. (2012). Mi plan, mi vida y mi futuro. Orientaciones pedagógicas para la Educación Económica y Financiera. Retrieved from http://www.mineducacion.gov.co/1621/a rticles-

343482_archivo_pdf_Orientaciones_Pe dag_Educ_Economica_y_Financiera.pdf

Principles, T. (2005). Recommendation on Principles and Good Practices for Financial Education and Awareness DIRECTORATE FOR FINANCIAL AND ENTERPRISE RECOMMENDATION OF THE COUNCIL, (July).

Reddy, R., Bruhn, M., \& Tan, C. (2013). Capacidades financieras en Colombia: Resultados de la encuesta nacional sobre comportamientos, actitudes y conocimientos financieros. Retrieved from

http://www.bancomundial.org/content/da $\mathrm{m}$ /Worldbank/document/LAC/Capacidad es Financieras en Colombia.pdf

Reyes Prialé, G., \& Yamada (2011). Encuesta de la Cultura Financiera en el Perú 2011 - Enfn - $2011 . \quad$ Lima. http://doi.org/ISBN:978-9972-57-185-5
Roa, M. (2010). Racionalidad, uso de información y decisiones financieras. Revistas CIDE, (478), 40. Retrieved from http://www.cide.edu/publicaciones/statu s/dts/DTE 478.pdf

Rodríguez, N. (2015). ¿Por qué nos peleamos por el dinero? Retrieved from http://www.elfinanciero.com.mx/misfinanzas/por-que-nos-peleamos-por-eldinero.html

Ruiz Ramirez, H. (2005). Conceptos sobre educación financiera. Observatorio de La Economía Latinoamericana, No 144, 20(ISSN 1696-8352), 1-10. Retrieved from

http://www.eumed.net/cursecon/ecolat/ $\mathrm{mx} / 2011 / \mathrm{hrr} . \mathrm{htm}$

Trump, D. J., \& Kiyosaki, R. T. (2007). Queremos que seas Rico.

Urbiola, A. (2011). Educación financiera en México. Participación de las entidades del sector ahorro y crédito popular. EstudiosSOCIALES8.Pmd; Secciones de Investigación, 202-217. Retrieved from

http://148.202.18.157/sitios/publicacione site/pperiod/estusoc/8/avance1.pdf

Visco, I. (2010). Educación financiera ante las repercusiones de la crisis. (Spanish). Boletín Del CEMLA, 56(3), 153-159. Retrieved from http://search.ebscohost.com/login.aspx? direct $=$ true $\& d b=a 9 h \& A N=55156104 \&$ lan $g=e s \&$ site $=$ ehost-live

White, E. G. (1890). Consejos Sobre Mayordomía Cristiana. 